\section{Organometallic Zinc Cages Open New \\ Possibilities for Semiconductors}

Spurred by the increasing use of nitride semiconductors such as $\mathrm{GaN}, \mathrm{AlN}$, and InGaN in optoelectronic, high-frequency, and highpower devices, there has been a lot of recent interest in finding single-source precursors to make these materials. Group 13 hydrazides are particularly attractive candidates for cleanly fabricating these important materials. However, not much is known about main-group hydrazides. In the February issue of Chemical Communications (DOI: 10.1039/ b516431a; p. 523), C. Redshaw from the University of East Anglia (Norwich, U.K.) and M.R.J. Elsegood from Loughborough University (Loughborough, U.K.) reported the preparation of novel tetrametallic zinc cages, which are good starting materials for the synthesis of other interesting architectures.

By reacting 1,1'-dimethylhydrazine with diethylzinc, the researchers were able to obtain a $\mathrm{Zn}_{4} \mathrm{~N}_{8}$ cage complex with two six-membered rings linked together by four five-membered rings. The lack of hydrogen bonding in these networks is expected to lead to improved conductivity for these materials. On reaction with tert-butyllithium, the cage-like structure of this novel hydrazide complex is disrupted, yielding a chain of $\mathrm{Zn}_{4} \mathrm{~N}_{7}$ clusters bridged together by lithium. The structure of the lithiated compound was determined using high-intensity $\mathrm{x}$-rays generated by the synchrotron at Daresbury Laboratory.

The researchers said that the $\mathrm{Zn}_{4} \mathrm{~N}_{8}$ organometallic "cube" can potentially be used to prepare many other interesting structural motifs that have not been seen before. These hydrazide complexes are expected to be useful precursors for the improved fabrication of semiconductors.

SARBAJIT BANERJEE

\section{Flexible Carbon Nanotube-Polymer Composites Display Excellent Electromechanical Properties}

Carbon nanotubes are known to exhibit exceptional electrical and mechanical properties. To tap these qualities for useful applications, nanotube structures need to be organized into stable device architectures. In the March 8 issue of Nano Letters (DOI: 10.1021/nl052238x; p. 413), Y.J. Jung of Northeastern University, S. Kar of Rensselaer Polytechnic Institute, A. Avadhanula of New Mexico State University, and their colleagues have presented a method to obtain aligned and patterned multiwalled carbon nanotubes (MWNTs) embedded in a soft poly (dimethylsiloxane) (PDMS) matrix. The resulting composite is an extremely flexible thin film with valuable electromechanical properties.

An array of cylindrical pillars (diameter, 500 $\mu \mathrm{m}$; height, 100 $\mu \mathrm{m})$ comprising vertically aligned MWNTs was grown on prepatterned $\mathrm{SiO}_{2} / \mathrm{Si}$ substrates using thermal chemical vapor deposition (CVD) of ferrocene and xylene at $800^{\circ} \mathrm{C}$. After pouring a prepolymer solution of PDMS on the substrate and curing at $100^{\circ} \mathrm{C}$ for $1 \mathrm{~h}$, self-standing nanotube-PDMS composite thin films were peeled off from the substrate. By varying the CVD growth conditions and changing the quantity of PDMS backbone, the shape and size of these composites can be easily controlled. Because of good wettability of MWNTs by PDMS, the films are relatively defect-free.

For systematic measurements of resistance, titanium wires were embedded into the matrix while it was being cured. It was observed that the resistance of these films increases linearly with both tensile and compressive strain, making them suitable for strain and pressure sensors. Also, since the conducting nature of the composite is retained under strain, the composites can be readily used in flexible electronic devices. This was demonstrated by field-emission measurements of a composite cathode fabricated by coating the thin film with Ti/Au. A field-enhancement factor of $\beta$ 19,100 was obtained, with a current density of $1 \mathrm{~mA} / \mathrm{cm}^{2}$ for a threshold field of $0.76 \mathrm{~V} / \mu \mathrm{m}$, which is extremely desirable for flexible field-emission devices.

TUSHAR PRASAD

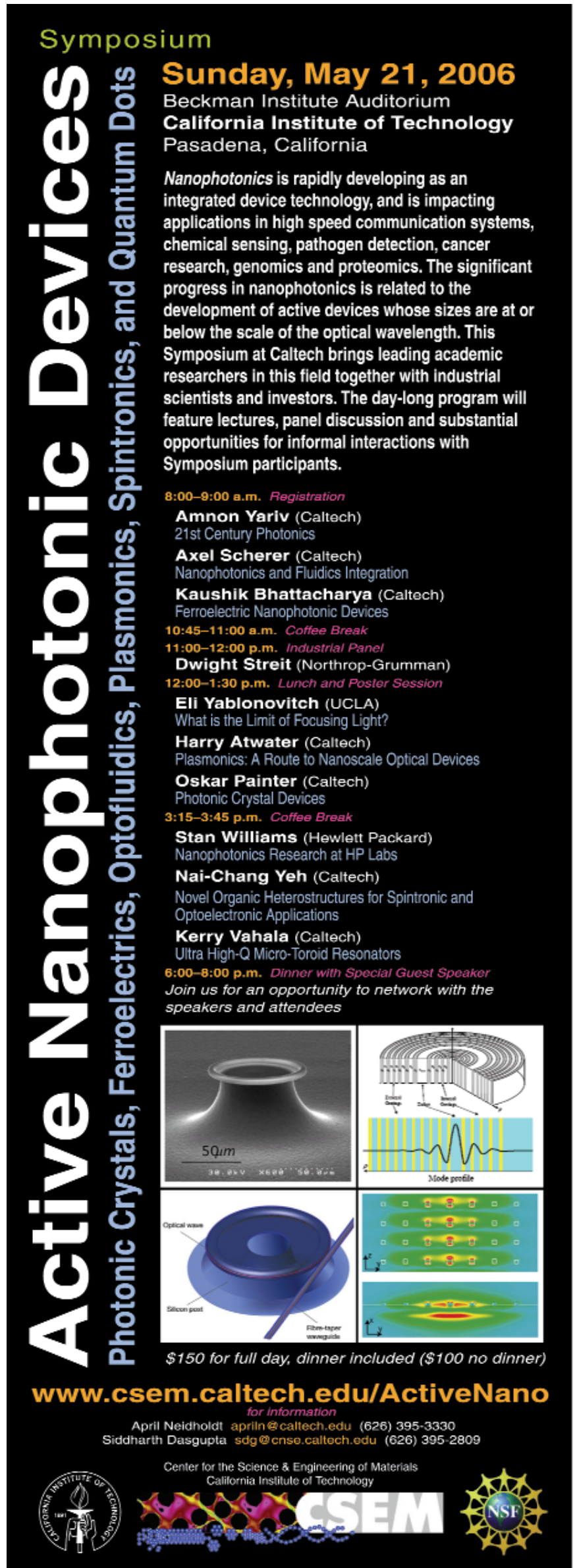

For more information, see http://www.mrs.org/bulletin_ads 